\title{
Bodyweight squats can induce post-activation performance enhancement on jumping performance: a brief report
}

\author{
Theodoros M. Bampouras a,* (D), Joseph I. Esformes b (D) \\ a Human Performance Laboratory, Lancaster Medical School, Lancaster University, Lancaster, LA1 4AT, UK. \\ b Cardiff School of Sport and Health Sciences, Cardiff Metropolitan University, Cardiff, CF23 6XD, UK. \\ *Corresponding author Email: t.bampouras@lancaster.ac.uk \\ DOI: https://doi.org/10.34256/ijpefs2044 \\ Received: 25-09-2020, Revised: 30-10-2020; Accepted: 31-10-2020; Published: 02-11-2020
}

\begin{abstract}
Post-activation potentiation enhancement (PAPE) refers to increased force generation following a muscular conditioning pre-activity that acutely enhances subsequent strength and power performance. Athlete apprehension to use heavy weights (i.e. $>80 \% 1 \mathrm{RM}$ ) immediately before a competition or inability to use weights before the performance (e.g. due to regulations) prevent materialising the benefits of PAPE. Therefore, this study examined whether PAPE can be induced with bodyweight squats. Sixteen healthy, team sports players (male: 10, female: 6 , mean \pm SD: age $22.2 \pm 3.0$ years, height $1.67 \pm 0.08 \mathrm{~m}$, body mass $70.2 \pm 8.2 \mathrm{~kg}$ ) performed three sets of ten repetitions of bodyweight squats with 30 seconds recovery between each set. A countermovement jump was performed 5 minutes before, 2 and 4 minutes after the squat sets and jump height was calculated. The results showed existence of PAPE with the jump height increasing at both $2(30.8 \pm 5.6 \mathrm{~cm}, \mathrm{p}=0.045, g=0.21)$ and $4(30.8 \pm 6.1 \mathrm{~cm}, \mathrm{p}=0.037, g=0.20)$ minutes, compared to baseline $(29.5 \pm 6.4 \mathrm{~cm})$. This is the first study to use bodyweight squats rather than loaded squats. Our findings indicate that three sets of ten repetitions of squats using bodyweight only can be a sufficient stimulus to induce PAPE.
\end{abstract}

Keywords: Conditioning stimulus, Countermovement jumps, Post-activation performance enhancement, Postactivation potentiation, Lower limb power

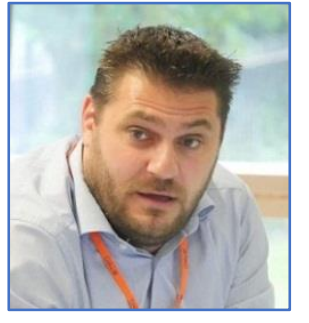

Dr. Theodoros M. Bampouras holds a PhD from Manchester Metropolitan University on the implications of muscle mechanics on muscle activation capacity assessment and he is currently a Lecturer in Biomechanics at Lancaster University, UK. Theo's research interests lie primarily in the areas of muscle performance, mechanics and function. He has published work in muscle function assessment methods and muscle conditioning for improved power, as well as in the areas of balance, gait and vision in healthy older adults. Additionally, he has published work examining the validity and reliability of assessment methods and equipment.

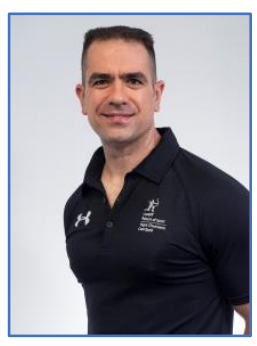

Dr Joseph Esformes has a Bachelor Degree in Sports Science and Physical Education from College of Sport Sciences (Athens, Greece), a Master Degree in Sport and Exercise Science from Manchester Metropolitan University, a Doctorate Degree in Exercise Physiology from the
University of Leeds, and has attained his Certification of Strength and Conditioning through the NSCA. Before embarking on an academic career, Joseph worked as a fitness instructor and strength coach for more than a decade. Since 2005, he has been a full-time University lecturer. Joseph is currently a Senior Lecturer in Strength and Conditioning at Cardiff Metropolitan University and a lecturer for Functional Movement Systems, delivering courses on the Functional Movement Screen (FMS) and corrective exercise. Previously, he taught Exercise Physiology at the University of Leeds and Sports Conditioning at the University of West of England. His research is in the areas of Strength and Conditioning and Cardiovascular Physiology. He has published in international peerreviewed journals and presented in international conferences in North America and Europe. 


\section{Introduction}

Contractile history influences muscular performance and its effect can result in fatigue (decreased neuromuscular force generation, [1]) or potentiation (enhanced force generation, [2]). Therefore, muscular performance following prior muscular activity (pre-activity) reflects the net balance between fatigue and potentiation. When fatigue has subsided, while potentiation remains, a pre-activity can increase muscular performance. This phenomenon is termed post-activation potentiation (PAP). It is traditionally confirmed using a series of evoked twitches, an evoked tetanic contraction or a sustained maximal voluntary contraction when examining the effect of a pre-activity on subsequent evoked muscle twitch force [3-5].

The practical relevance of this phenomenon in acutely enhancing athletic performance, by potentially improving strength and power parameters, has generated considerable research interest and ongoing debate [6-10]. As most of these studies focused on athletic enhancement, they explored the concept of post-activation performance enhancement (PAPE) rather than PAP. The majority of these studies have examined muscular performance enhancement after the typical time window PAP is expected to manifest (< 3 minutes $[11,12]$ ) and the presence of PAP was not verified. Such studies have examined different loads [8], exercise techniques [13] and modes [10], contractions $[14,15]$ and recovery intervals [9].

Regarding exercise intensity, typically heavier loads (i.e. $>80 \%$ of 1 repetition maximum; 1RM) have been used in studies investigating PAPE [6-10]. For example, Chiu et al., [6] used five parallel squat sets of one repetition at 90\% 1RM while Esformes et al., [10] used half-squat sets at 3RM, similar to Kilduff et al., [9]. However, studies have shown that PAPE can also be induced with lower loads $[7,8]$. The possibility that PAPE can be induced with lower loads could be an appealing notion to athletes who are apprehensive about lifting higher weights as part of their warm-up preparation before their competition performance. However, lower loads could still present a problem if additional equipment cannot be used before competition performance, either for practical or sport regulatory reasons; for example, a waiting area in swimming competitions. As any conditioning stimulus can potentially serve as a PAPE stimulus [16], the present study aimed to examine the effectiveness of bodyweight squats on PAPE, using countermovement jump (CMJ) height as the performance variable.

\section{Methods}

Sixteen healthy, team sports players (male: 10 , female: 6 , mean \pm SD: age $22.2 \pm 3.0$ years, height $1.67 \pm 0.08 \mathrm{~m}$, body mass $70.2 \pm 8.2 \mathrm{~kg}$ ) with a competitive playing experience of over four years but no specific resistance training experience, and with no injury in the year prior to testing volunteered to participate in the study.

Subjects were asked to refrain from eating two hours before testing and drinking coffee and alcohol 24 $\mathrm{h}$ before testing. Subjects were allowed to consume water ad libitum before and during the exercise task. Height was measured to the nearest $0.1 \mathrm{~cm}$ using a stadiometer (Holtain, Crymch, UK), while body mass was measured to the nearest $0.1 \mathrm{~kg}$ using a calibrated balance beam scale (Seca, Birmingham, UK).

Testing was completed with one visit to the laboratory. Subjects completed a standardised fiveminute warm-up, consisting of light-intensity cycling and stretching exercises for the quadriceps, hamstrings and triceps surae of both legs. For the quadriceps, the subjects pulled the ankle of the stretched leg and held it to maximum knee flexion. For the hamstrings, the heel of the stretched leg was planted on the ground and with the other leg further behind, the subject bent forwards towards the ankle of the stretched leg. Finally, for the triceps surae, the subjects with their stretched leg maintained straight leaned towards a wall. All stretches were performed once for $30 \mathrm{~s}$ (contralateral limb stretch duration) with $30 \mathrm{~s}$ rest between exercises, and held to the point of 'mild discomfort'. This approach was followed as it has been shown not to affect subsequent jumping performance [17]. A 2-min rest interval was allowed after the end of the warm-up. Subsequently, subjects performed three $\mathrm{CMJs}$, and the one with the highest jump height was used for further analysis (baseline CMJ; CMJBL). All jumps were performed with the hands on the hips throughout the movement to ensure isolation of power production to the lower limbs only [18]. The subjects were instructed to aim for maximum height, and the countermovement depth was self-selected.

Following a 5-min rest, the subjects executed three bodyweight parallel squat sets of 10 repetitions each, with $30 \mathrm{~s}$ rest between sets. The squat technique required subjects to descend until the inguinal fold was lower than the patella $\left(60^{\circ}-70^{\circ}\right.$ knee joint angle; fullextension $=180^{\circ}$ ). The squats started from an erect position, with the hands on the hips for the duration of the exercise, and were performed at a pace of 1 
second for each phase (i.e. 1 second lowering / 1 second rising), regulated by a metronome. A further 3 $\mathrm{CMJs}$ were performed at 2 (CMJ2) and 4 (CMJ4) minutes following the pre-activity, with the highest jump heights used for subsequent analysis. All countermovement jumps were performed using the OptoJump system, an optical measurement system consisting of two, $1 \mathrm{~m}$-long infrared photocell bars (with $1 \mathrm{~cm}$ intra-photocell spacing) recording at $1000 \mathrm{~Hz}$, with contact and flight time measured when the photocell beams were not interrupted and interrupted, respectively. Jump height was calculated using the flight time method [19]. The system has been reported as having high validity and reliability for these types of activities [20].

\subsection{Statistical analysis}

Data were checked for normality with the Shapiro-Wilk test and subsequently confirmed. A repeated-measures analysis of variance (ANOVA) was used to examine for any differences between CMJBL, $\mathrm{CMJ} 2$ and $\mathrm{CMJ} 4$. If a significant difference was found, pairwise comparisons between all three time points were conducted. Bonferroni correction was applied for the multiple pairwise comparisons, and the adjusted $p$ values are reported. Significance was set at 0.05 . For all statistical analysis, IBM SPSS v25.0 (Chicago, IL) was used. Finally, Hedges' $g$ was calculated as (mean difference between dataset1 and dataset2) / $\left.\operatorname{sqrt}\left(\left(\mathrm{SD}_{\text {dataset } 1}{ }^{2}+\mathrm{SD}_{\text {dataset2 }}{ }^{2}\right) / 2\right)\right)$ with $0.2,0.5$ and 0.8 interpreted as small, moderate and large effect, respectively [21]. Data are presented as mean \pm SD.

\section{Results}

Jump height had a significant main effect $\left(F_{2,30}\right.$ $=7.133, p=0.003)$. Pairwise comparisons revealed that CMLBL was significantly lower than CMJ2 by $4.3 \%$ ( $\mathrm{p}=0.045, g=0.21$ ) and by the same amount $(4.3 \%)$ to CMJ4 ( $p=0.037, g=0.20$ ). There was no difference between CMJ2 and CMJ4 ( $p=0.994)$ (Figure 1).

\section{Discussion}

The study aimed to examine the effect of bodyweight squats as a PAPE stimulus. Our findings indicate that three sets of ten repetitions of bodyweight-only squats can be a sufficient stimulus to induce PAPE. This is the first study to use bodyweight squats rather than loaded squats.

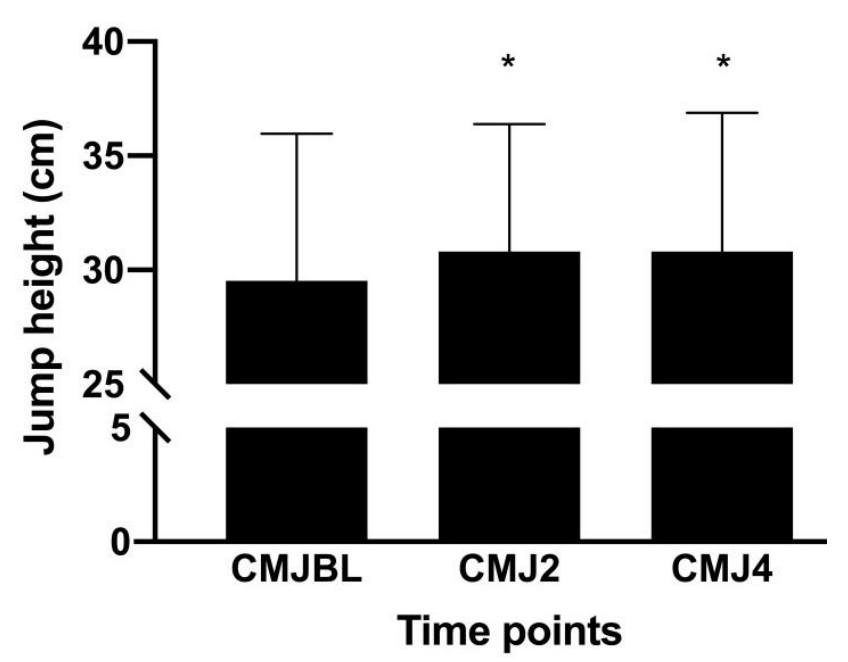

Figure 1 Mean countermovement jump height at baseline (CMJBL) and at 2 (CMJ2) and 4 (CMJ4) minutes, following bodyweight squats. Vertical bars denote SD. *denotes significant difference to CMJBL, $\mathrm{p}$ $<0.05$.

PAPE can have a considerable effect on athletic performance, as it can increase power production [16], an essential aspect in sports such as athletics [10]. Consequently, several studies have investigated how PAPE can be best induced to improve performance and has led to a variety of methods used; different loads $[7,8]$, activities [10], contractions $[14,15]$, technique [13] and rest intervals [9, 22].

Overall, studies report improved power performance, making PAPE an attractive training method for athletes seeking to improve power performance. However, the practice of lifting heavy weights as part of a pre-competition warm-up or pretrial preparation to induce PAPE may be challenging to implement. Competition restrictions may prevent access to, or use of weights during competitions (e.g. athletics, swimming), or the athletes may be apprehensive of their use before competition performance [10]. The ability to induce PAPE without any weight loading would remove these issues, while still offering the potential for improved performance. The novel findings of the present study show that this aim can be realised without the need for additional weight load, but by altering the conditioning stimulus volume through a decreased load (body mass) and increased repetitions. Further, the percentage of CMJ height improvement from the current study compares very favourably with several similar studies $[7,8,13]$. Thus, we posit that bodyweight alone can induce PAPE, therefore removing the need for any external weight to be used. 
Our findings are in agreement with previous studies that have typically used one set of three repetitions of weighted squats at $\sim 90 \% 1$ RM (e.g. [9, 10]. The fact that PAPE appears to be induced by both high intensity/low repetitions and low intensity/high repetitions suggests that one possible mechanism for inducing PAPE is not the intensity but the total work performed by the muscles. Indeed, Lowery et al., [22] found that acute jump performance and power were enhanced similarly by different protocols as long as total work was maintained the same. Taken together, these findings suggest that PAPE can be induced using different load/repetition combinations, as long as a sufficient volume of muscular work is performed to reach the 'threshold' required for PAPE to be induced [16].

A second possible mechanism for the induction of PAPE by the current protocol is the increased muscle temperature that would have been achieved by the repeated muscular contractions. An increased muscle temperature can increase the rate of force development [23], and an increased rate of force development can increase power performance [2] and is a determining factor for CMJ height [24]. Increased muscle temperature has also been suggested as a possible mechanism for PAPE, in that PAPE might reflect the effects of a typical warm-up rather than a potentiating effect [4]. The results of the present study do not support or reject the contribution of warming-up on PAPE. They do, however, offer support to the effect of PAPE as an additional performance enhancement method, since the improvements seen in the present study were achieved after the warm-up and therefore, in addition to any benefits that may have been achieved by the warm-up itself.

Finally, a third reason for these results could be the presence of PAP. Mitchell and Sale [25] found that 4 minutes after the completion of a 5RM squat, both an evoked twitch and a countermovement jump (performed on separate occasions) increased by $10.7 \%$ and $2.9 \%$, respectively. The study concluded that the increase seen in jump height was partially attributed to PAP. Given the exact time point with the present study and the somewhat smaller increase in jumping height seen in Mitchell and Sale's study, the possibility PAP contributed to our results cannot be discarded, albeit without verification of its presence.

The interaction of the rest interval and the load used is a factor to be considered for inducing PAPE [16]. Different loads require different rest intervals to be effective in achieving enhanced performance. As a result, various rest intervals have been examined, with Wilson et al., [26] showing $7 \mathrm{~min}$ to be the typical resting interval. The current study showed that peak PAPE can be induced as early as 2 minutes after the conditioning stimulus (bodyweight squats). As the load/rest interaction regulates PAPE, it is postulated that the lower neuromuscular fatigue caused by the bodyweight-only load pre-activity allowed PAPE to manifest earlier compared to higher intensity preactivities.

The current study used CMJ height as the only measure of performance, due to its relevance to athletic performance [27] and the high reliability to populations similar to ours [28]. Additionally, countermovement depth was self-selected and was not controlled. A change in muscle stiffness by the squat sets could have resulted in differences in countermovement depth, potentially impacting the jump height achieved [13]. However, visual inspection of the subjects' technique did not reveal any noticeable differences in countermovement depth during CMJBL, CMJ2 and CMJ4. Finally, we have attempted to address the lack of a control condition or group by the inclusion of effect sizes as part of the statistical analysis, to allow for a more informed interpretation of the results.

\section{Conclusions}

Previous studies have shown the effect of PAPE on power performance, using low to high percentages of $1 \mathrm{RM}$ squats as a conditioning stimulus. The present study is the first to demonstrate that bodyweight squats, using a higher repetition number, could also be sufficient for inducing PAPE. These findings can be of importance to sports where performance could be enhanced by improvement of short, explosive efforts but the use of equipment, such as free weights, is either prohibited or impractical. Bodyweight squats are easy to perform and do not require any equipment, thus allowing athletes to still benefit from PAPE. Further, even if the use of weights is possible, the use of a bodyweight-only pre-activity offers another alternative to the practitioners' arsenal for inducing PAPE earlier (i.e. at 2 minutes vs 7 minutes).

\section{References}

[1] D.E. Rassier, B.R. Macintosh, Coexistence of potentiation and fatigue in skeletal muscle, Brazilian Journal of Medical and Biological 
Research, 33(5) (2000) 499-508. DOI । PubMed

[2] N.A. Tillin, D. Bishop, Factors modulating postactivation potentiation and its effect on performance of subsequent explosive activities, Sports Medicine, 39(2) (2009)147-66. DOI । PubMed

[3] B. Requena, H. Gapeyeva, I. García, J. Ereline, M. Pääsuke, Twitch potentiation after vol-untary versus electrically induced isometric contractions in human knee extensor muscles, European Journal of Applied Physiology, 104(3) (2008) 463-472. DOI I PubMed

[4] B.R. Macintosh, M-E. Robillard, E.K. Tomaras, Should postactivation potentiation be the goal of your warm-up?, Applied Physiology, Nutrition, and Metabolism, 37(3) (2012) 546-550. DOI I PubMed

[5] C. Froyd, F.G. Beltrami, J. Jensen, T.D. Noakes, Potentiation increases peak twitch torque by enhancing rates of torque development and relaxation, Journal of Human Kinetics, 38 (2013) 83-94. DOI | PubMed

[6] L.Z.F. Chiu, A.C. Fry, L.W. Weiss, B.K. Schilling, L.E. Brown, S.L. Smith, Postactivation potentiation response in athletic and recreationally trained individuals, Journal of Strength and Conditioning Research, 17(4) (2003) 671-677. DOI I Pubmed

[7] V. Gourgoulis, N. Aggeloussis, P. Kasimatis, G. Mavromatis, A. Garas, Effect of a sub-maximal half-squats warm-up program on vertical jumping ability, Journal of Strength and Conditioning Research, 17(2) (2003) 342-344. DOI I PubMed

[8] I. Smilios, T. Pilianidis, K. Sotiropoulos, M. Antonakis, S.P. Tokmakidis, Short-term effects of selected exercise and load in contrast training on vertical jump performance, Journal of Strength and Conditioning Research, 19(1) (2005) 135139. DOI | PubMed

[9] L.P. Kilduff, H.R. Bevan, M.I.C. Kingsley, N.J. Owen, M.A. Bennett, P.J. Bunce, A.M. Hore, J.R. Maw, D.J. Cunningham, Postactivation potentiation in professional rugby players: optimal recovery, Journal of Strength and Conditioning Research, 21(4) (2007) 1134-1138. DOI | PubMed
[10] J.I. Esformes, N. Cameron, T.M. Bampouras, Postactivation potentiation following different modes of exercise, Journal of Strength and Conditioning Research, 24(7) (2010) 1911-1916. DOI | PubMed

[11] A.J. Blazevich, N. Babault, Post-activation potentiation versus post-activation perfor-mance enhancement in humans: historical perspective, underlying mechanisms, and current issues, Frontiers in Physiology, 10 (2019) 1359. DOI I PubMed

[12] O. Prieske, M. Behrens, H. Chaabene, U. Granacher, N.A. Maffiuletti, Time to differentiate postactivation "potentiation" from "performance enhancement" in the strength and conditioning community, Sports Medicine, 50 (2020) 15591565. DOI

[13] J.I. Esformes, T.M. Bampouras, Effect of back squat depth on lower-body postactivation potentiation Journal of Strength and Conditioning Research, 27(11) (2013) 2997-3000. DOI I PubMed

[14] K.P. Rixon, H.S. Lamont, M.G. Bemben, Influence of type of muscle contraction, gender, and lifting experience on postactivation potentiation performance, Journal of Strength and Conditioning Research, 21(2) (2007) 500505. DOI | PubMed

[15] J.I. Esformes, M. Keenan, J. Moody, T.M. Bampouras, Effect of different types of conditioning contraction on upper body postactivation potentiation, Journal of Strength and Conditioning Research, 25(1) (2011) 143-148. DOI | PubMed

[16] D.G. Sale, Postactivation potentiation: role in human performance, Exercise and Sport Sciences Reviews, 30(3) (2002) 138-143. DOI I PubMed

[17] S. Stafilidis, M. Tilp, Effects of short duration static stretching on jump performance, maximum voluntary contraction, and various mechanical and morphological parameters of the muscletendon unit of the lower extremities, European Journal of Applied Physiology, 115(3) (2015) 607-617. DOI | PubMed

[18] E.A. Harman, M.T. Rosenstein, P.N. Frykman, R.M. Rosenstein, The effects of arms and countermovement on vertical jumping, Medicine \& Science in Sports \& Exercise, 22(6) (1990) 825-833. DOI | PubMed 
[19] G.L. Moir, Three different methods of calculating vertical jump height from force plat-form data in men and women, Measurement in Physical Education and Exercise Science, 12(4) (2008) 207-218. DOI

[20] J.F. Glatthorn, S. Gouge, S. Nussbaumer, S. Stauffacher, F.M. Impellizzeri, N.A. Maffiuletti, Validity and reliability of Optojump photoelectric cells for estimating vertical jump height, Journal of Strength and Conditioning Research, 25(2) (2011) 556-560. DOI I PubMed

[21] C.O. Fritz, P.E. Morris, J.J. Richler, Effect size estimates: current use, calculations, and interpretation, Journal of Experimental Psychology: General, 141(1) (2012) 2-18. DOI I PubMed

[22] R.P. Lowery, N.M. Duncan, J.P. Loenneke, E.M. Sikorski, M.A. Naimo, L.E. Brown, F.G. Wilson, J.M. Wilson, The effects of potentiating stimuli intensity under varying rest periods on vertical jump performance and power, Journal of Strength and Conditioning Research, 26(12) (2012) 3320-3325. DOI I PubMed

[23] C.T. Davies, K. Young, Effect of temperature on the contractile properties and muscle power of triceps surae in humans, Journal of Applied Physiology, 55 (1983) 191-195. DOI I PubMed

[24] C.P. McLellan, D.I. Lovell, G.C. Gass, The role of rate of force development on vertical jump performance, Journal of Strength and Conditioning Research, 25(2) (2011) 379-385. DOI | PubMed

[25] C.J. Mitchell, D.G. Sale, Enhancement of jump performance after a 5-RM squat is associ-ated with postactivation potentiation, European Journal of Applied Physiology, 111 (8) (2011) 1957-1963. DOI | PubMed

[26] J.M. Wilson, N.M. Duncan, P.J. Marin, L.E. Brown, J.P. Loenneke, S.M.C. Wilson, E. Jo, R.P. Lowery, C. Ugrinowitsch, Meta-analysis of postactivation potentiation and power: effects of conditioning activity, volume, gender, rest periods, and training status, Journal of Strength and Conditioning Research, 27(3) (2013) 854859. DOI | PubMed

[27] G. Markovic, Does plyometric training improve vertical jump height? A meta-analytical review, British Journal of Sports Medicine, 41(6) (2007) 349-355. DOI I PubMed
[28] G. Markovic, D. Dizdar, I. Jukic, M. Cardinale, Reliability and factorial validity of squat and countermovement jump tests, Journal of Strength and Conditioning Research, 18(3) (2004) 551-555. DOI I PubMed

\section{Acknowledgement}

NIL

\section{Funding}

NIL.

\section{Authors Contribution}

Both authors contributed equally to study conceptualization, methodology, manuscript preparation, review and editing. Theodoros $M$. Bampouras collected the data. Both authors have read and approved the manuscript.

\section{Ethics Approval}

Approval was sought and granted by the Departmental Ethics Committee

\section{Conflict of interest}

The authors declare that they have no actual or potential conflict of interest, including financial, personal or other relationships with people or organizations that could have inappropriately influenced this work.

\section{Informed consent}

All participants gave written informed consent to participate in this study.

\section{About The License}

(c) The author(s) 2020. The text of this article is open access and licensed under a Creative Commons Attribution 4.0 International License 